\title{
Outcome of Superselective Bronchial Artery Embolization in Patients with Massive Hemoptysis
}

\author{
Jijo Oommen Roy, Sridevi Rajeeve, Anna Mathew ${ }^{1}$ \\ Departments of Pulmonology and 'Pharmacology, MOSC Medical College, Ernakulam, Kerala, India
}

\section{Abstract}

Hemoptysis, the coughing out of blood, is a fear-provoking symptom with possible life-threatening implications such as hypotensive shock, asphyxia and, myocardial stunning. The severity of hemoptysis can range from streaky episodes to massive episodes $(>500 \mathrm{ml} / \mathrm{day}$ or $>150$ $\mathrm{ml} /$ hour). The etiology of hemoptysis varies from lower respiratory causes such as tuberculosis, lung cancer, bronchiectasis, lung abscess, and pulmonary thromboembolism. The usual line of management is detailed history, imaging studies such as chest X-ray and CT thorax and excluding drugs affecting bleeding and clotting. Treating the underlying cause and including procoagulants usually helps to control moderate bleeding. Swift and effective management is important, especially in life-threatening cases as patients deteriorate fast. Bronchial artery embolization is indicated when conventional medical management fails and the patient is hemodynamically unstable to undergo emergency lobe/lung resection surgeries. We report our experience with four patients who presented to us with massive hemoptysis in whom superselective bronchial artery embolization (SBAE) was carried out successfully with gel-foam and coil. These patients were meticulously followed up for possible complications for one year.

Keywords: Gelfoam, massive hemoptysis, superselective bronchial artery embolization

\section{INTRODUCTION}

Hemoptysis, coughing out of blood, is a fear-provoking symptom with serious implications. The mortality rate is dependent on the magnitude of bleeding and the underlying etiology. The severity can range from streaky expectoration (mucus stained by blood) to massive episodes of hemoptysis, bringing up over 500 $\mathrm{ml} /$ day or $150 \mathrm{ml} / \mathrm{h}$ of blood. It is usually possible to control the symptoms in mild-to-moderate hemoptysis by actively treating the underlying cause and using procoagulants to control the bleeding. Massive hemoptysis is life-threatening because it has the potential to lead to hypotensive shock, asphyxia (due to coagulation of blood in the tracheobronchial tree), and myocardial stunning. Patients left untreated after a massive bout of hemoptysis have a mortality rate $>50 \%{ }^{[1]}$

The etiology of hemoptysis may vary from tuberculosis, lung cancer, bronchiectasis, and lung abscess to pulmonary thromboembolism. In a patient presenting with massive hemoptysis, the usual line of management is taking a

\begin{tabular}{|l|l|}
\hline \multicolumn{2}{|c|}{ Access this article online } \\
\hline Quick Response Code: & Website: \\
\hline & www.ijrc.in \\
\cline { 2 - 2 } & \\
\hline
\end{tabular}

swift history including the details of drugs taken such as anticoagulants or antiplatelet drugs followed by a thorough clinical examination. Investigations to rule out bleeding and clotting disorders should be done. Imaging of the chest with an X-ray and computerized tomography (CT) scan will help localize the site of the lesion. As the most important source of hemoptysis is the bronchial artery, it is essential to visualize the bronchial circulation before any intervention is undertaken; hence, angiography of the pulmonary vascular tree is a prerequisite to bronchial embolization.

Embolization of the bronchial artery is indicated when conventional medical management fails and surgical

Address for correspondence: Dr. Anna Mathew, Department of Pharmacology, MOSC Medical College, Kolenchery, Ernakulam - 682 311, Kerala, India. E-mail: mosc.research@gmail.com

This is an open access journal, and articles are distributed under the terms of the Creative Commons Attribution-NonCommercial-ShareAlike 4.0 License, which allows others to remix, tweak, and build upon the work non-commercially, as long as appropriate credit is given and the new creations are licensed under the identical terms.

For reprints contact: WKHLRPMedknow_reprints@wolterskluwer.com

How to cite this article: Roy JO, Rajeeve S, Mathew A. Outcome of superselective bronchial artery embolization in patients with massive hemoptysis. Indian J Respir Care 2021;10:107-11.

Received: 24-04-2020

Accepted: 07-09-2020 Published: 31-01-2021 
intervention is not tenable, as when the patient is hemodynamically unstable to undergo lung resection surgery.

The first superselective embolization of the bronchial artery to control hemoptysis was performed in 1973 by Rémy et al. ${ }^{[2]}$ The same group reported a large series of 104 patients, in 1977, where embolization was performed in the bronchial and non-bronchial arteries to control massive hemoptysis. Of these, 49 were treated during active hemoptysis and immediate control of hemorrhage was possible in 41 (84\%) of these patients. $^{[3]}$

Tanaka et al. were able to document enhanced control of hemoptysis while using a microcatheter to perform the superselective bronchial artery embolization (SBAE) compared to selective catheterization of bronchial artery. ${ }^{[4]}$ The use of microcatheters in a coaxial technique is now used in most interventions with proven efficacy.

Ishikawa et al., in a study published in 2017, performed SBAE in 489 patients. They reported a success rate of $93.4 \%$ and a complication rate of $1.6 \%$. There were 57 cases of relapse of hemoptysis and 23 deaths in the observed group. ${ }^{[5]}$

Another study, published in 2017 by Panda et al., reported a technical success rate varying between $81 \%$ and $100 \%$ with superselective BAE. The embolizing agent used was polyvinyl alcohol (PVA) particles. They noted a recurrence rate that varied between $12 \%$ and $57 \%$, while the incidence of complications noted was $0.1 \%{ }^{[6]}$

The aim of the embolization is to terminate the blood flowing to the vessels that are feeding the bleeding in the lung, while the blood flow to the surrounding areas is preserved. Several agents have been used for embolization such as PVA particles, gelatin sponge particles, gelfoam pledgets or slurry, thrombin, and glue. The most commonly used PVA particles provide permanent occlusion, while gelatin sponge particles are said to provide only temporary occlusion. The recommended particle size for agents used for embolization is $>200-250 \mu \mathrm{m}$ as smaller size particles have a greater risk of embolizing the spinal artery and causing tissue ischemia and neurologic damage. PVA particles have been proven to give good results when used in the particle size range of 300-500 $\mu \mathrm{m} \cdot{ }^{[7-12]}$

In this article, we report our experience with four patients who presented with spontaneous massive hemoptysis to this rural tertiary care teaching hospital in Kerala. In each of these patients, we performed bronchial arteriography followed by superselective embolization of the bronchial artery with PVA particles in the recommended range.

\section{Subjects and Methods}

This study was done on patients who presented to our emergency room with acute, spontaneous massive hemoptysis during 2013-2014. In our study, we defined hemoptysis as bleeding originating in the lower respiratory tract referring to the area below the level of the lower border of cricoid cartilage. ${ }^{[13]}$
Patient-1 was a 41-year-old male patient who had active tuberculosis at the time of presentation, while Patient- 2 was an 88-year old female patient who had infective exacerbation of bronchiectasis. Patient-3 who had a microaneurysm in the left upper lobe was a 46-year old male patient and Patient-4 who was 66 years old had necrotizing consolidation. After collection of historical and sociodemographical details and clinical examination, laboratory investigations were done to rule out bleeding disorders, and chest radiography and imaging with CT scan were performed to localize the lesion and determine etiology. An aortogram was performed to visualize the bronchial circulation and identify the arteries feeding the bleed. The images obtained were stored on a compact disc as angiographic runs.

Under local anesthesia and strict aseptic precautions, the right common femoral artery was percutaneously punctured in all the patients, and a $6 \mathrm{~F}$ RDC Guiding introduction sheath was inserted followed by a 4F Glide catheter. Selective aortograms were performed to identify the abnormal/bleeding vessels, in each patient. The site of bleeding was thus localized, so that only distal vessels at the site were embolized and much of the proximal blood supply was preserved. In three of the four patients, SBAE was performed using two $\mathrm{ml}$ of PVA particles of size $300 \mathrm{mg}$ to $500 \mathrm{mg}$ using a coaxial microcatheter system. Coil (Cook) embolization was carried out in Patient-4. Check angiograms were performed in all patients after SBAE to ensure arrest of bleeding.

After the procedure, three patients (Patients 1, 2, and 3) were stable and were managed in the ward. Patient-4 had to be maintained on invasive mechanical ventilation in the intensive care for three days, before being weaned off successfully. He was given blood transfusions, intravenous vasopressors, and other general measures for correcting the hypovolemic shock. The patient was also initiated on appropriate antitubercular therapy according to the Directly Observed Treatment Short course regimen after his condition became stable.

\section{RESULTS}

Three of our study subjects were men aged 41,46 , and 66 years, respectively, and one was an 88-year-old female. The mean age of the four patients was 60.25 years, with the youngest patient being 41 years and the oldest patient being 88 years.

All three men in the study had an active smoking history with a mean smoking habit of five to ten cigarettes a day. The etiology of the hemoptysis was readily identifiable in all the cases. Patient-1 was a newly diagnosed smear-positive pulmonary tuberculosis patient whose radiograph revealed a right upper lobe cavity with surrounding consolidation. Patient- 2 was an 88-year-old female patient where infective exacerbation of a long-standing bronchiectasis was determined to be the inciting cause of hemoptysis. The 46-year-old man, Patient-3, gave a history of childhood surgical ASD closure as the only salient surgical history. The cause of his bleed was a microaneurysm fed by the left bronchial artery. Patient- 4 was a 66 -year-old male patient, in whom the instigating cause of hemoptysis was 
determined to be a necrotizing consolidation of the right upper lobe. Two of the patients had undergone earlier evaluation by bronchoscopy as well for locating the anatomic site of bleeding.

All the patients reported to the emergency room with first episodes of spontaneous acute onset of coughing out copious amounts of blood. The median quantity of blood expectorated was $600 \mathrm{ml}$ (range $350-1000 \mathrm{ml}$ ). There was no history of trauma to chest or intake of anticoagulants.

The vital signs of Patients-1, 2, and, 3 were within normal limits. Patient-4 presented to the ER in a state of deteriorating hypotensive shock and hence he was shifted to the intensive care department for close monitoring and resuscitation with IV fluids and vasopressors. A preliminary chest radiograph, CT scan, and aortogram were performed to help identify the bleeding lobe. None of our patients received blood transfusions prior to the procedure.

Selective aortograms were performed to identify the abnormal/bleeding vessels, in each patient, as seen in Figures 1a, 2a, 3a, and 4a.

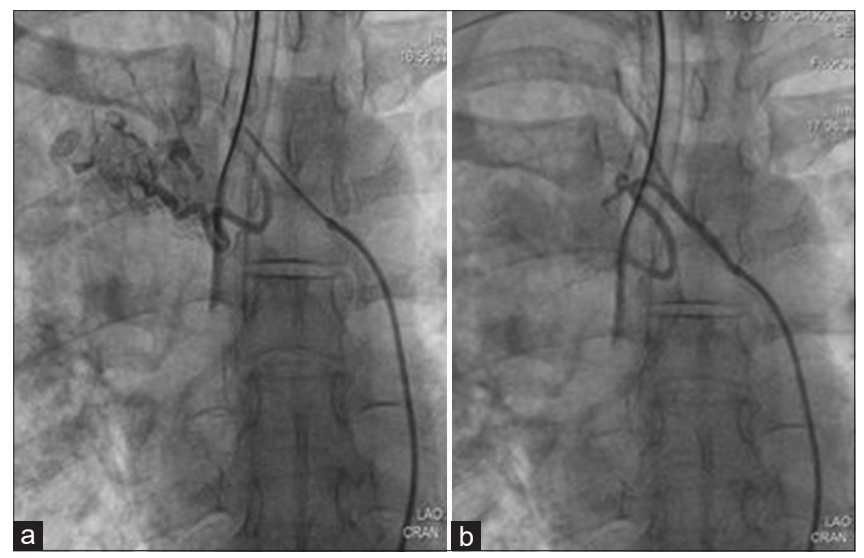

Figure 1: (a) Patient-1: Preembolization image showing active bleeding in a pulmonary tuberculosis cavity in right upper lobe. (b) Patient-1: Postembolization image showing arrested bleeding and diminished blood flow to the region

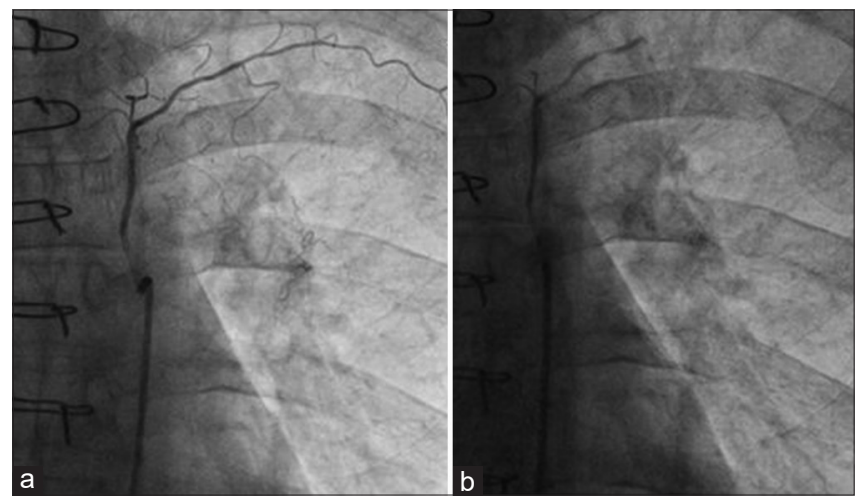

Figure 3: (a) Patient-3: Preembolization image showing active bleeding in a microaneurysm fed by left bronchial artery. (b) Patient-3: Postembolization image showing lack of blood flow to the distal blood vessels
The SBAE was performed in each of the patients. After embolization of the targeted vessels to stasis or near stasis, a reduction in the size of the bronchial arteries is a common finding. Immediate control of the bleeding was achieved in all patients putting the hemoptysis control rate at $100 \%$. Follow-up check angiograms were performed after the SABE to ensure arrest bleeding. The check angiogram revealed successful embolization of bleeding vessels in all four patients as seen in Figures 1b, 2b, 3b, and 4b.

There were no episodes of recurrence of hemoptysis. All patients, except Patient- 4 who had presented in shock, were hemodynamically stable after the intervention. Patient-4 was weaned off mechanical ventilation and vasopressors in 3 days.

A follow-up procedure for recurrence was not warranted in any case. The postoperative period was uneventful. There were no procedure-related complications in any patient. All patients were discharged within a week with a median period of hospital stay of 4 days.

The patients were all meticulously followed up every month for a year for any complication.

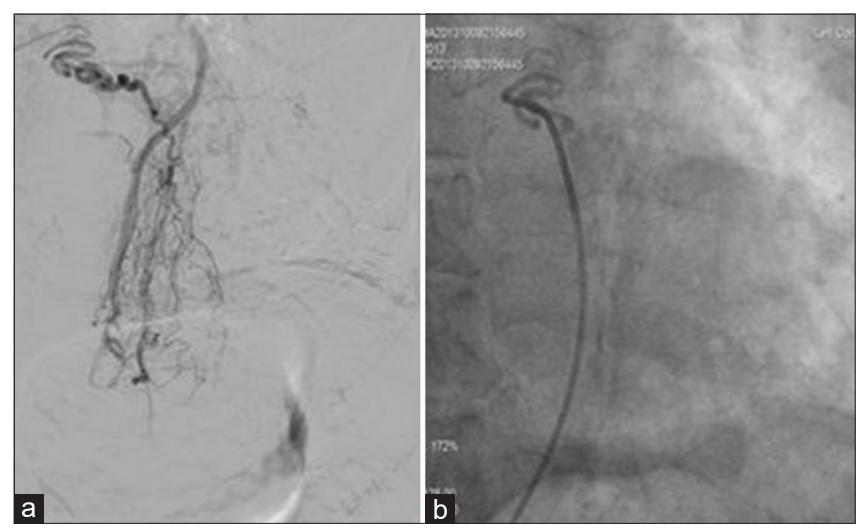

Figure 2: (a) Patient-2: Preembolization image showing active bleeding in a bronchiectatic left upper lobe. (b) Patient-2: Postembolization image showing diminished blood flow to the distal blood vessels

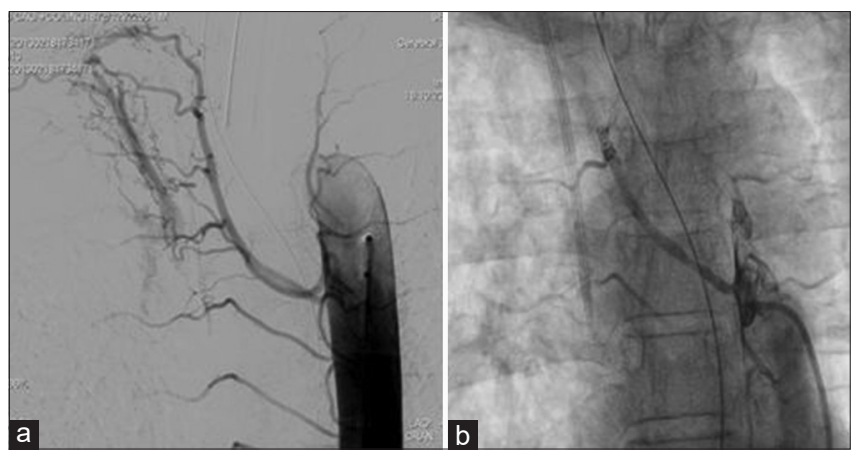

Figure 4: (a) Patient-4: Preembolization image showing active bleeding in a necrotizing consolidation of right middle lobe. (b) Patient-4: Postembolization image showing diminished blood flow to the region and control of bleeding 


\section{Discussion}

Evaluation of the patient prior to bronchial angiography is essential for localizing the lesion and establishing the etiology. Patients with hemoptysis usually undergo chest roentgenography, CT scanning of the thorax, and bronchoscopy. Of these, the CT scan of the chest has been reported to be superior to bronchoscopy in the evaluation of hemoptysis. ${ }^{[14]}$ Hirshberg et al. documented that used alone, a chest $\mathrm{CT}$ scan, was the most sensitive diagnostic test, correctly identifying $67 \%$ of patients. If CT scanning was to be combined with bronchoscopy, it would be possible to correctly diagnose $93 \%$ of patients. ${ }^{[15]}$ Preembolization CT Aortogram with bronchoscopy aids in accurately narrowing down the site of bleeding in most cases. In this way, much of the proximal blood supply is preserved and the distal vessels are embolized when the SBAE is performed.

We performed a chest radiograph, thoracic CT scan, and arteriogram in all the patients. In the early days of bronchial arteriography, there were reports of complications such as transverse myelitis due to the use of nonionic contrast media, which was neurotoxic. This has diminished over the years with less toxic agents being available. In addition, two of our patients also underwent a bronchoscopic assessment for locating the anatomic site of the bleeding.

We used PVA particles and coils for the embolization and there was immediate control of bleeding in all four patients (100\%). This is similar to a study published by Cremaschi et al., who had assessed 209 patients embolized for hemoptysis and of these 205 (98\%) achieved immediate control of bleeding. ${ }^{[16]}$

Another complication during embolization was the inadvertent embolization of the spinal arteries. Superselective embolization of bronchial arteries (SBAE) was developed to prevent this complication. SBAE refers to embolization of the more terminal branches of the arterial tree, beyond the origin of the spinal arteries. It is also important to embolize nonbronchial systemic arteries if they are angiographically shown to be contributing to the blood supply of the area.

Recurrent rebleed despite adequate embolization has been quoted as a significant complication ranging from $9 \%$ to $29 \%$ after embolization. ${ }^{[16-21]}$ None of our patients developed a rebleed during the immediate postprocedure period or during the 1 year of follow-up. It is also important to identify the etiology and treat the underlying pulmonary process to decrease the vascularity of the region and the development of vascular collaterals.

Other complications encountered include subintimal dissection due to catheter insertion, perforation caused by the guidewire, and reflux of embolic agents into the aorta. Some of the symptoms that may be manifested include transient pleuritic chest pain, shoulder pain, dysphagia, and hematoma in the groin. ${ }^{[22]}$

Ramakantan et al. evaluated the results of SBAE in 140 patients with tuberculosis and observed that twelve patients developed post-procedure complications including transient left orbital/forehead pain, transient paraparesis, and transient dysphagia. ${ }^{[20]}$ These complications occurred despite post-SBAE arteriography showing good selective catheterization of the bronchial artery. None of our patients experienced any neurologic sequelae, transient or permanent, and all have made good recovery of general health.

Mal et al. described three major types of spinal cord complications, which occurred despite good, selective catheterization of the bronchial artery. They include Brown-Sequard's syndrome, which regressed after four months without sequelae; paraparesis with spontaneous regression after two weeks; and complete paraplegia without regression. ${ }^{[17]}$ In our study, none of the patients experienced any neurologic sequelae, transient or permanent, following SBAE and all have now made good recovery of general health.

The availability of preembolization CT scan and aortogram with bronchoscopy has made SBAE safe and accurate by enabling visualization of the bleeding site. The site of bleeding is thus narrowed down, so that only distal vessels need to be embolized and most of the proximal blood supply can be preserved.

Although conventionally, definitive treatment for massive hemoptysis is surgery, SBAE may help treat patients where surgery is untenable. Our experience of employing SBAE for control of massive hemoptysis has shown that this minimally invasive intervention is highly efficacious in controlling massive hemoptysis.

\section{ConcLusion}

Superselective Bronchial Artery Embolization is a highly efficacious minimally invasive procedure for controlling massive haemoptysis.

\section{Declaration of patient consent}

The authors certify that they have obtained all appropriate patient consent forms. In the form, the legal guardian has given his consent for images and other clinical information to be reported in the journal. The guardian understands that names and initials will not be published and due efforts will be made to conceal identity, but anonymity cannot be guaranteed.

\section{Acknowledgments}

The authors are grateful to the Management of MOSC Medical College for all the resources provided. We fully acknowledge the contribution of the interventional cardiologists who performed the procedure, the lab staff for all the technical support, and the Department of Cardiology at MOSC Medical College, Kolenchery, for the images provided.

\section{Financial support and sponsorship}

Nil.

\section{Conflicts of interest}

There are no conflicts of interest. 
Roy, et al:: Superslective bronchial artery embolization

\section{RefEREnCES}

1. Jean-Baptiste E. Clinical assessment and management of massive hemoptysis. Crit Care Med 2000;28:1642-7.

2. Rémy J, Voisin C, Dupuis C, Beguery P, Tonnel AB, Denies JL, et al. Treatment of hemoptysis by embolization of the systemic circulation. Ann Radiol (Paris) 1974;17:5-16.

3. Rémy J, Arnaud A, Fardou H, Giraud R, Voisin C. Treatment of hemoptysis by embolization of bronchial arteries. Radiology 1977; 122:33-7.

4. Tanaka N, Yamakado K, Murashima S, Takeda K, Matsumura K, Nakagawa $\mathrm{T}$, et al. Superselective bronchial artery embolization for hemoptysis with a coaxial microcatheter system. J Vasc Interv Radiol 1997;8:65-70.

5. Ishikawa $\mathrm{H}$, Hara M, Ryuge M, Takafuji J, Youmoto M, Akira M, et al. Efficacy and safety of super selective bronchial artery coil embolisation for haemoptysis: a single-centre retrospective observational study. BMJ Open 2017;7: 014805.

6. Panda A, Bhalla AS, Goyal A. Bronchial artery embolization in hemoptysis: A systematic review. Diagn Interv Radiol 2017;23:307-17.

7. Cohen AM, Doershuk CF, Stern RC. Bronchial artery embolization to control hemoptysis in cystic fibrosis. Radiology 1990;175:401-5.

8. Fellows KE, Khaw KT, Schuster S, Shwachman H. Bronchial artery embolization in cystic fibrosis; technique and long-term results. J Pediatr 1979;95:959-63.

9. Bookstein JJ, Moser KM, Kalafer ME, Higgins CB, Davis GB, James WS. The role of bronchial arteriography and therapeutic embolization in hemoptysis. Chest 1977;72:658-61.

10. Muthuswamy PP, Akbik F, Franklin C, Spigos D, Barker WL. Management of major or massive hemoptysis in active pulmonary tuberculosis by bronchial arterial embolization. Chest 1987;92:77-82.

11. Vujic I, Pyle R, Hungerford GD, Griffin CN. Angiography and therapeutic blockade in the control of hemoptysis. The importance of nonbronchial systemic arteries. Radiology 1982;143:19-23.
12. van Heesch HA, Tjan GT, Lampmann LE. Treatment of hemoptysis by embolization of the systemic arteries with isobutyl-2-cyanoacrylate: Technique and long term results. J Interv Radiol 1988;3:63-8.

13. Fraser RG, Pare JAP, Pare PD, Fraser RS, Genereux G. Diagnosis of diseases of the chest, 3rd ed. By Philadelphia: W.B. Saunders Co., 1988, 394-6.

14. McGuinness G, Beacher JR, Harkin TJ, Garay SM, Rom WN, Naidich DP. Hemoptysis: Prospective high-resolution CT/bronchoscopic correlation. Chest 1994;105:1155-62.

15. Hirshberg B, Biran I, Glazer M, Kramer MR. Hemoptysis: Etiology, evaluation, and outcome in a tertiary referral hospital. Chest 1997;112:440-4.

16. Cremaschi P, Nascimbene C, Vitulo P, Catanese C, Rota L, Barazzoni GC, et al. Therapeutic embolization of bronchial artery: A successful treatment in 209 cases of relapse hemoptysis. Angiology 1993;44:295-9.

17. Mal H, Rullon I, Mellot F, Brugière O, Sleiman C, Menu Y, et al. Immediate and long-term results of bronchial artery embolization for life-threatening hemoptysis. Chest 1999;115:996-1001.

18. Rabkin JE, Astafjev VI, Gothman LN, Grigorjev YG. Transcatheter embolization in the management of pulmonary hemorrhage. Radiology 1987; $163: 361-5$

19. Hayakawa K, Tanaka F, Torizuka T, Mitsumori M, Okuno Y, Matsui A, et al. Bronchial artery embolization for hemoptysis: Immediate and long-term results. Cardiovasc Intervent Radiol 1992;15:154-8.

20. Ramakantan R, Bandekar VG, Gandhi MS, Aulakh BG, Deshmukh HL. Massive hemoptysis due to pulmonary tuberculosis: Control with bronchial artery embolization. Radiology 1996;200:691-4.

21. Uflacker R, Kaemmerer A, Picon PD, Rizzon CF, Neves CM, Oliveir ES, et al. Bronchial artery embolization in the management of hemoptysis: Technical aspects and long-term results. Radiology $1985 ; 157: 637-44$

22. Swanson KL, Johnson CM, Prakash UB, McKusick MA, Andrews JC, Stanson AW. Bronchial artery embolization: Experience with 54 patients. Chest 2002;121:789-95. 